\title{
Determining the value of TB active case-finding: current evidence and methodological considerations
}

\author{
H. Sohn ${ }^{1}$, S. Sweeney ${ }^{2}$, D. Mudzengi ${ }^{3}$, J. Creswell ${ }^{4}$, N. A. Menzies ${ }^{5}$, G. J. Fox ${ }^{6,7}$, P. \\ MacPherson ${ }^{8,9,10}$, D. W. Dowdy ${ }^{1}$ \\ ${ }^{1}$ Department of Epidemiology, Johns Hopkins Bloomberg School of Public Health, Baltimore, MD, \\ USA \\ ${ }^{2}$ London School of Hygiene \& Tropical Medicine, London, UK \\ ${ }^{3}$ The Aurum Institute, Johannesburg, South Africa \\ ${ }^{4}$ The Stop TB Partnership, UNOPS, Geneva, Switzerland \\ ${ }^{5}$ Department of Global Health and Population, Harvard T H Chan School of Public Health, \\ Boston, MA, USA \\ ${ }^{6}$ Sydney Medical School, Faculty of Medicine and Health, The University of Sydney, Sydney, \\ NSW, Australia \\ ${ }^{7}$ Woolcock Institute of Medical Research, Glebe, NSW, Australia \\ ${ }^{8}$ Department of Clinical Sciences, Liverpool School of Tropical Medicine, Liverpool, UK \\ ${ }^{9}$ Malawi-Liverpool-Wellcome Trust Clinical Research Programme, Malawi \\ ${ }^{10}$ Clinical Research Department, London School of Hygiene \& Tropical Medicine, London, UK
}

\section{SUMMARY}

\begin{abstract}
Active case-finding (ACF) is an important component of the End TB Strategy. However, ACF is resource-intensive, and the economics of ACF are not well-understood. Data on the costs of ACF are limited, with little consistency in the units and methods used to estimate and report costs. Mathematical models to forecast the long-term effects of ACF require empirical measurements of the yield, timing and costs of case detection. Pragmatic trials offer an opportunity to assess the cost-effectiveness of ACF interventions within a 'real-world' context. However, such analyses generally require early introduction of economic evaluations to enable prospective data collection on resource requirements. Closing the global case-detection gap will require substantial additional resources, including continued investment in innovative technologies. Research is essential to the optimal implementation, cost-effectiveness, and affordability of ACF in high-burden settings. To assess the value of ACF, we must prioritize the collection of high-quality data regarding costs and effectiveness, and link those data to analytical models that are adapted to local settings.
\end{abstract}

Correspondence to: Hojoon Sohn, Johns Hopkins Bloomberg School of Public Health, 615 N Wolfe Street E6531, Baltimore, MD 21205, USA. hsohn6@jhu.edu.

Conflict of interests: none declared. 


\section{Keywords}

tuberculosis; ACF; health economics; modelling; screening

With more than three million people with incident TB not notified to public health authorities each year, closing the global case detection gap is essential to achieving the post-2015 End TB targets. ${ }^{1-3}$ Advances in diagnostic testing have improved case detection but have not effected major reductions in TB incidence and mortality. ${ }^{4-6}$ Health systems delays and barriers to accessing TB care remain common. ${ }^{7,8}$

Active case-finding (ACF) - that is, efforts to screen for TB in target populations (e.g., by geography and risk groups) outside of routine health services-may help to close the case-detection gap by linking patients to TB care early in their disease course. ${ }^{9}$ ACF interventions vary considerably in their locations, screening algorithms and target populations. Approaches include community-based door-to-door symptom screening, household contact investigation, and interventions using mobile technologies and diagnostic solutions to enhance TB case detection/linkage to care. However, the population-level impact of ACF remains uncertain. One of the most comprehensive trials of ACF (the ZAMSTAR trial) found limited impact on the population prevalence of TB. ${ }^{10}$ Another trial in Zimbabwe (DETECTB) highlighted the importance of effective implementation to achieve population-level impact. ${ }^{11}$ Recent modeling studies ${ }^{12,13}$ and pragmatic trials in Viet $\mathrm{Nam}^{14,15}$ have reinvigorated interest in ACF, suggesting that ACF can effectively reduce TB prevalence.

A key criticism of ACF is its resource-intensive nature. Model-based economic evaluations have suggested that ACF could be cost-effective under many conditions. ${ }^{16-19}$ However, the process by which ACF might be integrated into existing health systems, the incremental costs of such integration, and the corresponding epidemiological and economic value (i.e., return on investment) remain largely unknown. With global funding efforts such as the Stop TB Partnership's TB REACH initiative ${ }^{20}$ and the Global Fund to Fight AIDS, Tuberculosis and Malaria creating opportunities for technological and process innovation, economic evidence is needed to support decisions regarding strategic adoption and scale-up of ACF interventions. Here, we discuss the current state of evidence regarding the economics of $\mathrm{ACF}$ and consider future priorities in this field.

\section{DECISION-MAKERS' PERSPECTIVE}

For optimal and efficient resource allocation decisions, evidence on cost-effectiveness and affordability is critical. ${ }^{21}$ An evidence-based approach to policymaking and strategic intervention implementation is particularly important in high TB burden countries where resources and infrastructure remain heavily reliant on external donors. ${ }^{22}$ For resourceintensive and programmatically complex interventions such as ACF, understanding incremental benefits and costs is challenging. "Cost per TB case detected" may be the most intuitive metric of cost-effectiveness, but the relationship between cases detected and lives saved (or disability-adjusted life years [DALYs] averted) is not straightforward. ${ }^{19}$ More recently the additionality concept (i.e., increase in TB notifications above recent 
trends) has been used to estimate programmatic and health outcomes (e.g., incremental TB cases detected or deaths averted) and population-level impact (e.g., incidence and prevalence). ${ }^{23}$ Summary indicators of health impact (e.g., DALYs) ${ }^{24}$ are not always useful in evaluating ACF interventions, as high-quality data linking ACF to DALYs averted are scarce, approaches to ACF are heterogeneous, and methods of evaluation are potentially biased (e.g., before-and-after evaluations incorporate temporal trends).

Along with the Global Fund, the TB REACH initiative has been a major funding source for ACF and other interventions to increase TB notifications. TB REACH has funded 313 projects in 54 countries (totaling USD155 million) across seven "waves" since 2010. TB REACH initially recommended a cost ceiling of USD350 per additional case detected, building on a previous initiative (FIDELIS) that used a criterion of USD80 per additional treatment success during DOTS expansion. ${ }^{25}$ The first TB REACH funding cycle showed that, while projects were generally successful in increasing notifications, the average cost per additional notification was much higher (USD864) ${ }^{26}$ By its fourth wave of funding, TB REACH abandoned the USD350 ceiling with an understanding that ACF costs vary greatly by setting, operationalization, and human resource costs. Furthermore, the cost per additional case detected may not fully reflect the benefits of reduced community transmission, and short-term assessments may not accurately estimate the long-term value of ACF interventions. ${ }^{16}$ Thus, generating evidence on optimizing the implementation and scale-up of cost-effective ACF interventions that can be affordable and sustained (e.g., broad-based scale-up vs. periodic targeted campaigns) is a high priority.

\section{EVALUATING COSTS OF ACTIVE CASE-FINDING INTERVENTIONS}

The Table shows published evidence on the unit costs of ACF, as collated by the Global Health Cost Consortium (GHCC) and a scoping review of the current literature. ${ }^{17,27-40}$ Interventions range from community-based door-to-door symptom screening to mobile diagnostic solutions (e.g., mobile vans equipped with X-ray and/or GeneXpert ${ }^{\circledR}$ MTB/RIF machines [Cepheid, Sunnyvale, CA, USA]). Existing cost estimates vary widely, reflecting variations in ACF modalities, TB prevalence, application of technologies, operationalization of ACF interventions across different settings, and the types of data, analytic methods and perspectives used for cost analyses.

Determinants of ACF intervention costs often vary substantially by unit and modality. For example, the cost per person screened will reflect the size of the target population, underlying TB prevalence, and method of screening. In household contact investigations, for example, multiple follow-up visits or alternative ways of accessing contacts may be necessary to optimize yield. ${ }^{41-44}$ As a contrasting example, screening in correctional facilities can be operationally more efficient. However, screening a large number of inmates requires logistical planning, caution to protect participants' rights, and appropriate linkage to treatment after release. It is therefore important to evaluate the specific operational and contextual factors influencing the resource implications of each individual ACF intervention; primary data collection is often necessary. 
The Table also illustrates that outcomes of screening are reported inconsistently. For example, "yield" may be reported as the number of confirmed cases detected per person tested or per index case. Methods used to estimate and report costs also vary considerably. The appropriate reporting unit may differ by intended policy application. For example, the unit cost per person screened may be useful for evaluating operational efficiency or for planning and budgeting, whereas the cost per TB case identified and/or completing treatment may be more useful for modeling cost-effectiveness. The incremental yield of case-finding and the corresponding impact on TB incidence are two of the most critical outcomes; however, these can be difficult to measure. Another important metric of impact is the reduction in diagnostic delay. In the context of routine care, these outcomes can only be estimated using assumptions about the counter-factual scenario (of no screening). Consistency in the reporting of economic outcomes is needed to facilitate reconciliation of estimates across studies. To address these concerns, the GHCC Reference Case for Estimating the Costs for Global Health Services and Interventions ${ }^{45}$ recommends two main 'units' for reporting unit costs of ACF interventions: cost per person screened and cost per person diagnosed with TB.

As described in the Reference Case, the process for estimating the cost of ACF includes estimating the 'unit' cost for services such as outpatient visits and diagnostic tests, and then multiplying by the quantities utilized (Figure 1). ACF poses unique challenges for cost data collection. Depending on the form of ACF and activities involved, costs may be observed above the service level (particularly for community-based interventions) ${ }^{27}$ or outside of the health system entirely (e.g., correctional facilities ${ }^{40}$ ) — for which costing methods are less well defined. ${ }^{39,40}$ Depending on the ACF modality and accessibility, it may be difficult for researchers to observe services directly, making the estimation of staff time and equipment use difficult. Outreach workers may be apprehensive about completing timesheets or could modify their behavior when observed, thereby distorting costs. Embedding data collectors in outreach teams and/or using mobile devices to estimate time allocation could improve the quality of cost data.

When unit costs are not estimable with reasonable accuracy, a top-down costing approach using an activity-based analytic framework may be used. ${ }^{31}$ This approach compartmentalizes costs by major ACF activity categories: pre-implementation, screening, diagnosis, and treatment support. Main cost outcomes include total program costs, service unit costs (e.g., cost per Xpert test), and cost per program yield (e.g., cost per patient screened, patient tested, TB diagnosis, and treatment completion). Top-down costing may be less precise and portrays different types of costs than a bottom-up approach (typically resulting in higher cost estimates) ${ }^{46,47}$ but this framework allows cost data to be disaggregated in a standardized and comparative manner. Prospective data collection can enable estimation of uncertainties in cost estimates associated with program operations and workloads.

Finally, TB impose substantial costs on households as patients seek care for diagnosis and treatment. ${ }^{48}$ Early case detection by ACF interventions may help to address these costs by reducing diagnostic attempts and delays in treatment. ${ }^{49}$ Two studies have demonstrated that households of patients identified through ACF were less likely to experience catastrophic 
costs - defined as excessive costs resulting in adverse financial coping and increased risk of adverse treatment outcome ${ }^{50}$ — due to TB illness. ${ }^{35,51}$ As many high-burden countries pursue innovative policies to reduce catastrophic TB-related costs, more research is needed to investigate the mechanisms and impact of ACF interventions on household-incurred costs.

\section{EVALUATING THE COST-EFFECTIVENESS OF ACTIVE CASE-FINDING USING EPIDEMIOLOGICAL MODELS}

In deciding whether the costs of ACF interventions are justified, they must be evaluated against health outcomes, including 1) better health for individuals with TB who are identified through ACF, 2) harms produced by incorrect TB diagnoses and treatment for individuals without $\mathrm{TB}$, and 3 ) reductions in $\mathrm{TB}$ transmission due to earlier diagnosis. Mathematical modelling is commonly used to quantitatively estimate each of these outcomes, including attendant uncertainty. Such models use systems of equations to represent relationships between observed outcomes (such as TB case notifications and effect sizes from empirical trials) and outcomes of interest (such as incremental differences in long-term survival produced by an intervention). These models can estimate outcomes that are difficult or impossible to measure empirically. ${ }^{52,53}$

For the first outcome (health benefits for individuals with TB), models typically assume that ACF will identify individuals earlier in the course of TB disease. Historically, models have represented TB disease as a series of discrete health states, such as LTBI and untreated active TB. ${ }^{54}$ Earlier case detection can be represented as a higher exit rate from the untreated TB state, producing a proportional reduction in the cumulative hazard of TB mortality. However, this approach may overestimate the reductions in TB morbidity and mortality associated with ACF interventions, as increased symptomatology is likely correlated with both elevated TB-related mortality and health-seeking behavior. Thus, ACF may identify a larger fraction of individuals with asymptomatic or early TB relative to passive case detection, resulting in a smaller-than-expected reduction in TB mortality. The converse may be true if ACF is targeted to communities with poor healthcare access, which may identify many individuals with advanced TB. ${ }^{55}$ Recent analyses provide more granular descriptions of TB disease progression and associated patterns of healthcare seeking, ${ }^{16,19,56,57}$ including stratified analyses among key population subgroups undergoing $\mathrm{ACF}^{26}$

The second outcome (harms produced by incorrect TB diagnoses and treatment) is a major concern in modelling the impact of ACF interventions, given the lower prevalence of TB among screened populations and the imperfect specificity of TB diagnostics. Representing the possibility of false-positive diagnoses in TB models requires an estimate of the overall specificity of the ACF diagnostic algorithm. These estimates involve substantial uncertainty, as the observed fraction testing positive reflects both the underlying prevalence of TB and the accuracy of the diagnostic algorithm. ${ }^{58}$ Moreover, while TB models provide a mechanistic description of the process generating new TB cases, there is typically no matching model for symptomatic individuals without TB who are treated empirically. Despite these challenges, addressing the uncertainty around false-positive diagnoses is preferable to ignoring the associated costs and harms, ${ }^{59}$ as both health systems and patients 
incur substantial costs (e.g., unnecessary treatment and out-of-pocket costs) and adverse consequences following false-positive diagnoses. Models therefore ideally use outcomes that can incorporate false-positive diagnoses (e.g., DALYs averted rather than changes in TB incidence), assess costs from a broader societal perspective, ${ }^{60}$ and explore the impact of false-positive diagnoses in sensitivity analysis.

The third outcome (changes in secondary TB cases) requires a population-level model to predict the reduction in Mycobacterium tuberculosis transmission generated by earlier disease detection and the resulting reduction in incident TB disease. This process requires assumptions about how an individual's infectiousness and the susceptibility of their contacts change over time. ${ }^{61}$ While individual infectiousness may increase with more advanced disease, contact networks may become "saturated" over time, reducing incremental susceptibility to ongoing exposure. ${ }^{62}$ The net effect of earlier case detection through ACF on reducing transmission is therefore uncertain. More sophisticated models could explore the impact of these competing mechanisms. Models must also consider the additional value of targeting groups with higher transmission potential (high bacillary load, high numbers of respiratory contacts, increased contact with HIV-positive individuals and infants). The transmission effects of ACF interventions are delayed relative to direct effects and may vary according to program coverage (i.e., relative reach of the intervention in the target population). Thus, analyses that incorporate transmission effects must assess outcomes over a sufficiently long timeframe for reductions in TB incidence to occur.

\section{EVALUATING THE COST-EFFECTIVENESS OF ACTIVE CASE-FINDING EMPIRICALLY WITHIN TRIALS}

Pragmatic trials allow the cost-effectiveness of ACF interventions to be evaluated empirically within a 'real-world' context. One recent cluster randomized trial evaluating ACF among household contacts in Viet Nam provides an illustration. ${ }^{63}$ The study intervention involved screening household contacts of patients with TB four times over 2 years, comparing notification rates among contacts against those who were not screened. The study incorporated in-trial costs for patient assessment, travel supplementation and diagnostic tests. Treatment costs were based upon a national costing survey. ${ }^{64}$ The estimated cost of the intervention was USD544 (95\% CI \$330-\$1375) per DALY averted, which was deemed highly cost-effective in this context, in comparison to routine passive case-finding.

Figure 2 provides a simplified schematic of the processes involved in conducting a costeffectiveness study within a pragmatic trial. Such analyses have important advantages. Both costs and effectiveness are measured in the same population and collected prospectively using standardized questionnaires, participant diaries and schedules of routine costs. This increases the accuracy and robustness of estimates, as prospective measurement reduces the likelihood of omitting important resource-use data (e.g., costs of unscheduled visits, additional tests and transportation costs for sputum samples). Measurement of patient costs in trial settings also enables estimates of between-patient variability, providing more precise estimates of uncertainty than is possible in simulation studies which must assume nonempiric distributions for outcomes and costs. Performing costing studies within trials further 
allows calculation of costs in the control/standard-of-care arm. For example, patients who do not receive ACF may experience higher catastrophic $\operatorname{costs}^{35}$ due to delayed diagnosis, which may have important implications for cost-effectiveness from the patient and societal perspective.

An additional advantage of prospective measurement is the ability to quantify costs and resource use that may only become apparent during the process of implementing and operationalizing an ACF intervention (Figure 3). This is particularly important as the costs to implement and sustain ACF interventions are not well characterized. When collected, such evidence can inform resource needs for continued (and/or expanded) implementation, including oversight and monitoring and evaluation, once research-related resources are withdrawn. ${ }^{65}$ An increasing number of pragmatic trials are embedding economic evaluations; these studies will provide important information about the costeffectiveness of ACF interventions as implemented in different contexts. ${ }^{15,66,67}$ Continued data collection during the scale-up (regional and/or nationwide) of ACF interventions would allow re-evaluation of cost-effectiveness based on 'real-world' data during and after scale-up and can further characterize important economic barriers to scale-up that may only emerge after research studies have concluded.

Although economic evaluations embedded within pragmatic trials have important advantages, there are several important challenges. First, collection of detailed patient costs, such as using cost diaries, can be time-consuming and complex, ${ }^{68}$ creating potential barriers to recruitment and/or missing data. While capturing detailed costs in a specific context improves the precision of cost-effectiveness estimates, costs may not generalize to other settings. For this reason, sensitivity analyses are critical to inform policymakers in other contexts. Furthermore, cost-effectiveness estimates are dependent on the statistical power of the main trial to detect differences in (cost and effectiveness) outcomes. For example, the ZAMSTAR (Zambia/South Africa TB and AIDS Reduction) study estimated an 18\% reduction in TB prevalence in the community-wide household TB screening arm, but this did not meet conventional thresholds for statistical significance. ${ }^{10}$ Hence, the ability to perform a corresponding cost-effectiveness analysis with prevalence as the main outcome was impaired. Similar considerations can arise if cost data collection is underpowered (e.g., if only a sub-sample of participants are included for costing). For the advantages of trial-embedded economic evaluations to be realized, these challenges must be addressed.

\section{NOVEL TECHNOLOGIES AND PROCESS INNOVATIONS}

In recent years, numerous technological and process innovations have emerged that could improve operational efficiency, equity, effectiveness, and value of ACF interventions (Figure 4). These developments may transform how ACF programs are operationalized and how their value and impact are appraised.

Geospatial mapping of community risk factors such as poverty, crowding, air quality, access to healthcare, and demographics (e.g., male-to-female ratio, age distribution) can be combined with local geo-referenced TB data to build spatiotemporally explicit models to target ACF more efficiently. ${ }^{69-71}$ Such approaches can improve operational efficiency and 
yield by moving from targeting geographically defined populations (cities, districts, regions) or groups of people who share common risk factors for TB disease (HIV-positive people, prisoners, contacts of TB cases, miners, homeless people $)^{72,73}$ to prioritizing screening of neighborhood-level hotspots identified by surveillance of TB case notifications. ${ }^{74} \mathrm{In}$ this approach, local TB surveillance data (case notification rates, estimated prevalence of undiagnosed TB) would be combined with neighborhood-level data on TB risk factors (e.g., prevalence of HIV, ART coverage, poverty, diabetes) ${ }^{75}$ and indicators of health access. As many diseases of public health importance (HIV, hypertension, diabetes) share population distributions with $\mathrm{TB},{ }^{76-78}$ this would allow local public health authorities to target ACF interventions to where the yield and benefit are likely to be greatest. Improved targeting of ACF interventions can also help integrate programmatic components that can result in improve cost/resource-sharing of operational infrastructure (e.g., prevention, screening and linkage-to-care programs for other health conditions), ultimately improving operational efficiency, cost-effectiveness, and sustainability of TB ACF.

Symptom enquiry and chest X-ray have been the cornerstones of ACF screening for decades. ${ }^{79}$ However, symptoms such as cough are common in resource-limited settings, ${ }^{80}$ and have low sensitivity and specificity for $\mathrm{TB} .{ }^{81}$ Furthermore, traditional analog chest $\mathrm{X}$-ray requires expensive and non-durable equipment, trained and motivated readers, and an effective data archiving system. ${ }^{82}$ Newer robust and highly-mobile digital X-ray machines are now widely available and likely to be cost-saving over their lifetime use, particularly if also employed for non-TB conditions. ${ }^{83}$ Picture archiving and communication systems (PACS) and telemedicine reading services further enhance implementation feasibility. Computer-aided reading/interpretation (CAR/I) X-ray systems are as accurate as expert radiologists, ${ }^{84}$ rapid $(<1 \mathrm{~min})$, and can be run on a local computer without internet access. Evaluating the cost and cost-effectiveness of implementing highly portable X-ray systems with CAR/I in high-burden settings is therefore an important research priority.

Improvements in screening methods and target populations alone may not close the gap in case detection. Positive results from initial screening must often be confirmed with a highly specific diagnostic test. ${ }^{85}$ GeneXpert testing (using either Xpert MTB/RIF or Xpert Ultra cartridges) has improved bacteriologic diagnosis, ${ }^{86,87}$ but only a limited number of ACF projects have demonstrated cost-effectiveness and operational feasibility. ${ }^{31,88}$ Pooling samples from multiple patients into a single Xpert cartridge may reduce the costs of ACF interventions, and recent proof-of-concept studies have demonstrated promising performance. ${ }^{89,90}$ New mobile diagnostic tests (e.g., GeneXpert Omni and Edge) and sample transport devices (e.g., drones) may improve access to ACF, reducing diagnostic delay and expanding coverage to hard-to-reach areas (e.g., mountainous regions or settings without proper road networks). Prior to scale-up, however, optimization of screening interventions in each local context will be essential. Policy makers must ensure that interventions are acceptable, feasible, affordable and cost-effective from the perspective of the health system. ${ }^{6}$

\section{CONCLUSION}

Closing the global case detection gap will require additional resources and continued investment in innovative technology and research to ensure optimal implementation, cost- 
effectiveness, and affordability in high-burden settings. With global emphasis on "no person left behind" and universal health coverage, we must identify ways to focus ACF activities toward reaching marginalized populations who are often missed by routine services. Doing so has important economic implications, with potential for both increased costs and increased health benefits. Technological and process innovations for ACF have the potential to address these challenges; however, such interventions may not be economically or operationally viable at scale unless they can benefit from economies of scale and scope. To effectively evaluate interventions for ACF in the End TB era, we must emphasize collection of high-quality data on costs and effectiveness, linkage of those data to appropriate analytical models, incorporation of novel technologies and process innovations, and contextualization of evidence to a variety of local settings.

\section{Acknowledgements}

This work was made possible by the generous support from National Institutes of Health/National Institute of Allergy and Infectious Diseases (R01AI146555 to NAM), the Australian National Health and Medical Research Council Career Development Fellowship (APP1148372 to GJF), and the Wellcome Trust (206575/Z/17/Z to PM).

\section{References}

1. World Health Organisation. Global tuberculosis report, 2019. WHO/CDS/TB/2019. 15. Geneva, Switzerland: WHO, 2019.

2. World Health Organization. The End TB Strategy. Geneva, Switzerland: WHO, 2014.

3. Stop TB Partnership. The Paradigm Shift: Global Plan to End TB: 2018-2022. Geneva, Switzerland: WHO, 2019.

4. Theron G, et al. Feasibility, accuracy, and clinical effect of point-of-care Xpert MTB/RIF testing for tuberculosis in primary-care settings in Africa: A multicentre, randomised, controlled trial. Lancet 2014; 383: 424-435. [PubMed: 24176144]

5. Menzies NA, et al. Population health impact and cost-effectiveness of tuberculosis diagnosis with Xpert MTB/RIF: a dynamic simulation and economic evaluation. PLoS Med 2012; 9:11; e1001347. [PubMed: 23185139]

6. Sohn $\mathrm{H}$, et al. Informing decision-making for universal access to quality tuberculosis diagnosis in India: an economic-epidemiological model. BMC Med 2019; 17: 155. [PubMed: 31382959]

7. Getnet F, et al. Delay in diagnosis of pulmonary tuberculosis in low-and middle-income settings: systematic review and meta-analysis. BMC Pulm Med 2017; 17: 1-15. [PubMed: 28049457]

8. Cox H, et al. Delays and loss to follow-up before treatment of drug-resistant tuberculosis following implementation of Xpert MTB/RIF in South Africa: a retrospective cohort study. PLoS Med 2017; 14: 1-19.

9. Golub JE, et al. Active case-finding of tuberculosis: historical perspective and future prospects. Int J Tuberc Lung Dis 2005; 9: 1183-1203. [PubMed: 16333924]

10. Ayles H, et al. Effect of household and community interventions on the burden of tuberculosis in southern Africa: the ZAMSTAR community-randomised trial. Lancet 2013; 382: 1183-1194. [PubMed: 23915882]

11. Corbett EL, et al. Comparison of two active case-finding strategies for community-based diagnosis of symptomatic smear-positive tuberculosis and control of infectious tuberculosis in Harare, Zimbabwe (DETECTB): a cluster-randomised trial. Lancet 2010; 376: 1244-1253. [PubMed: 20923715]

12. Houben RMGJ, et al. Feasibility of achieving the 2025 WHO global tuberculosis targets in South Africa, China, and India: a combined analysis of 11 mathematical models. Lancet Glob Heal 2016; 4: e806-e815

13. Vesga JF, et al. Assessing tuberculosis control priorities in high-burden settings: a modelling approach. Lancet Glob Health 2019; 7: e585-e595. [PubMed: 30904521] 
14. Fox GJ, et al. Household-contact investigation for detection of tuberculosis in Vietnam. N Engl J Med 2018; 378: 221-229. [PubMed: 29342390]

15. Marks GB, et al. Community-wide screening for tuberculosis in a high-prevalence setting. N Engl J Med 2019; 381: 1347-1357. [PubMed: 31577876]

16. Azman AS, et al. How much is tuberculosis screening worth? Estimating the value of active case-finding for tuberculosis in South Africa, China, and India. BMC Med 2014; 12: 1-9.

17. Yadav RP, et al. Cost-effectiveness of a tuberculosis active case-finding program targeting household and neighborhood contacts in Cambodia. Am J Trop Med Hyg 2014; 90: 866-872. [PubMed: 24615134]

18. Shah L, et al. Cost-effectiveness of active case-finding of household contacts of pulmonary tuberculosis patients in a low HIV, tuberculosis-endemic urban area of Lima, Peru. Epidemiol Infect 2017; 145:6; 1107-1117 [PubMed: 28162099]

19. Johnson K, Uhlig E, Jo Y, Creswell J, Qin ZZ, Dowdy DW, and Sohn H. Incremental value of tuberculosis active case-finding in the context of symptom-associated care seeking. 2020. [Manuscript in Preparation]

20. Stop TB Partnership. TB REACH. Geneva, Switzerland: Stop TB Partnership, 2020. http:// www.stoptb.org/global/awards/tbreach/. Accessed April 2020.

21. Cromwell I, et al. Real-world health care priority setting using explicit decision criteria: a systematic review of the literature. BMC Health Serv Res 2015; 15: 1-11. [PubMed: 25603697]

22. World Health Organization. Global investments in Tuberculosis research and development: past, present and future. A policy paper prepared for the first WHO global ministerial conference on ending tuberculosis in the sustainable development era: a multisectoral response. Geneva, Switzerland: WHO, 2017.

23. Blok L, et al. A pragmatic approach to measuring, monitoring and evaluating interventions for improved tuberculosis case detection. Int Health 2014; 6(3): 181-188. [PubMed: 25100402]

24. Baltussen RMP, et al. Making choices in health: WHO guide to cost-effectiveness analysis. Geneva, Switzerland: World Health Organization, 2003.

25. Rusen ID, et al. FIDELIS - Innovative approaches to increasing global case detection of tuberculosis. Am J Public Health 2006; 96: 14-16. [PubMed: 16317206]

26. Creswell J, et al. A multi-site evaluation of innovative approaches to increase tuberculosis case notification : summary results. PLoS One 2014; 9(4): e94465. [PubMed: 24722399]

27. Atif M, et al. Tracing contacts of TB patients in Malaysia: costs and practicality. Springerplus 2012; 1: 1-8. [PubMed: 23984219]

28. Sohn H, et al. Costs and operation management of community outreach program for tuberculosis in tribal populations in India. Public Health Action 2019; 9(2): 58-62. [PubMed: 31417854]

29. Sohn $\mathrm{H}$, et al. Cost-effectiveness of contact screening strategies for tuberculosis among high-school adolescents in South Korea. Int J Tuberc Lung Dis 2018; 22: 496-503. [PubMed: 29663953]

30. James R, et al. Tuberculosis active case-finding in Cambodia: A pragmatic, cost-effectiveness comparison of three implementation models. BMC Infect Dis 2017; 17: 1-7. [PubMed: 28049444]

31. Jo Y, et al. Standardized framework for evaluating costs of active case-finding programs: an analysis of two programs in Cambodia and Tajikistan. PLoS One 2020; 15: e0228216. [PubMed: 31986183]

32. Kranzer K, et al. Feasibility, yield, and cost of active tuberculosis case finding linked to a mobile HIV service in Cape Town, South Africa: a cross-sectional study. PLoS Med 2012; 9: 1-11.

33. Chihota VN, et al. Liquid vs. solid culture for tuberculosis: performance and cost in a resourceconstrained setting. Int J Tuberc Lung Dis 2010; 14: 1024-1031. [PubMed: 20626948]

34. Eang MT, et al. Early detection of tuberculosis through community-based active case-finding in Cambodia. BMC Public Health 2012; 12: 1. [PubMed: 22214479]

35. Muniyandi M, et al. Catastrophic costs due to tuberculosis in South India: comparison between active and passive case finding. Trans R Soc Trop Med Hyg 2019; 114:3; 185-192.

36. Sekandi JN, et al. Cost-effectiveness analysis of community active case-finding and household contact Investigation for tuberculosis case detection in urban Africa. PLoS One 2015; 10: 1-18. 
37. Steffen RE, et al. Cost-effectiveness of Quantiferon®-TB Gold-In-Tube versus tuberculin skin testing for contact screening and treatment of latent tuberculosis infection in Brazil. PLoS One 2013; 8: 1-8.

38. Vassall A, et al. Reforming tuberculosis control in Ukraine: results of pilot projects and implications for the national scale-up of DOTS. Health Policy Plan 2009; 24: 55-62. [PubMed: 19056804]

39. Winetsky DE, et al. Screening and rapid molecular diagnosis of tuberculosis in prisons in Russia and Eastern Europe: a cost-effectiveness analysis. PLoS Med 2012; 9:11; e1001348. [PubMed: 23209384]

40. Zishiri V, et al. Implementing a large-scale systematic tuberculosis screening program in correctional facilities in South Africa. Open Forum Infect Dis 2015; 2: ofu121 [PubMed: 25884008]

41. Deery CB, et al. A home tracing program for contacts of people with tuberculosis or HIV and patients lost to care. Int J Tuberc Lung Dis 2014; 18: 534-540. [PubMed: 24903789]

42. Velen K, et al. Household HIV testing uptake among contacts of TB patients in South Africa. PLoS One 2016; 11:5; e0155688. [PubMed: 27195957]

43. Kigozi NG, et al. Yield of systematic household contact investigation for tuberculosis in a highburden metropolitan district of South Africa. BMC Public Health 2019; 19:1; 867. [PubMed: 31269950]

44. Armstrong-Hough M, et al. Drop-out from the tuberculosis contact investigation cascade in a routine public health setting in urban Uganda: a prospective, multi-center study. PLoS One 2017; 12:11; e0187145. [PubMed: 29108007]

45. Vassall A, et al. Reference case for estimating the costs of global health services and interventions. London, UK: LSHTM Research Online, 2017. https://researchonline.1shtm.ac.uk/id/ eprint/4653001/ Accessed January 2020.

46. Cunnama L, et al. Using top-down and bottom-up costing approaches in LMICs: the case for using both to assess the incremental costs of new technologies at scale. Health Econ 2016; 19: 1300-1317.

47. Tucker A, et al. Redefining and revisiting cost estimates of routine ART care in Zambia: an analysis of ten clinics. J Int AIDS Soc 2020; 23: e25431. [PubMed: 32064766]

48. Barter DM, et al. Tuberculosis and poverty: the contribution of patient costs in sub-Saharan Africa. A systematic review. BMC Public Health 2012; 12:1; 980. [PubMed: 23150901]

49. Lorent $\mathrm{N}$, et al. Community-based active tuberculosis case finding in poor urban settlements of Phnom Penh, Cambodia: a feasible and effective strategy. PLoS One 2014; 9: e92754. [PubMed: 24675985]

50. Lönnroth K, et al. Beyond UHC: monitoring health and social protection coverage in the context of tuberculosis care and prevention. PLoS Med 2014; 11:9; e1001693. [PubMed: 25243782]

51. Gurung SC, et al. The role of active case-finding in reducing patient incurred catastrophic costs for tuberculosis in Nepal. Infect Dis Poverty 2019; 8:1; 99. [PubMed: 31791412]

52. World Health Organization. Guidance for country-level TB modelling. Geneva, Switzerland: WHO, 2018.

53. Zwerling A, et al. Mathematical modelling and tuberculosis: advances in diagnostics and novel therapies. Adv Med 2015; 2015: 1-10.

54. Houben RMGJ, et al. Spotting the old foe-revisiting the case definition for TB. Lancet Respir Med 2019; 7: 199-201. [PubMed: 30823971]

55. Mugwagwa T, et al. Comparing different technologies for active TB case-finding among the homeless: a transmission-dynamic modelling study. Sci Rep 2018; 8:1; 1-12. [PubMed: 29311619]

56. Kasaie P, et al. Timing of tuberculosis transmission and the impact of household contact tracing: an agent-based simulation model. Am J Respir Crit Care Med 2014; 189: 845-852. [PubMed: 24559425]

57. Ahmad RRA, et al. How to optimize tuberculosis case finding: explorations for Indonesia with a health system model. BMC Infect Dis 2009; 9:1; 87. [PubMed: 19505296] 
58. Lalli M, et al. Investigating the impact of TB case-detection strategies and the consequences of false positive diagnosis through mathematical modelling. BMC Infect Dis 2018; 18:1; 340. [PubMed: 30031378]

59. Houben RMGJ, et al. What if they don't have tuberculosis? The consequences and trade-offs involved in false-positive diagnoses of tuberculosis. Clin Infect Dis 2018; 68:1; 150-156.

60. Wilkinson $\mathrm{T}$, et al. The international decision support initiative reference case for economic evaluation: an aid to thought. Value Health 2016; 19: 921-928. [PubMed: 27987641]

61. Turner RD, et al. Tuberculosis infectiousness and host susceptibility. J Infect Dis 2017; 216(Suppl): S636-S643. [PubMed: 29112746]

62. Dowdy DW, et al. Is passive diagnosis enough? The impact of subclinical disease on diagnostic strategies for tuberculosis. Am J Respir Crit Care Med 2013; 187(5): 543-551. [PubMed: 23262515]

63. Lung T, et al. Household contact investigation for the detection of tuberculosis in Vietnam: economic evaluation of a cluster-randomised trial. Lancet Glob Heal 2019; 7: e376-e384.

64. Nhung NV, et al. Measuring catastrophic costs due to tuberculosis in Viet Nam. Int J Tuberc Lung Dis 2018; 22: 983-990. [PubMed: 30092862]

65. Sohn $\mathrm{H}$, et al. The costs of implementing health interventions in resource-limited settings: ignored at our own peril. Implementation Sci 2020; 15: 86.

66. Pooran A, et al. Point of care Xpert MTB/RIF versus smear microscopy for tuberculosis diagnosis in southern African primary care clinics: a multicentre economic evaluation. Lancet Glob Heal 2019; 7: e798-e807.

67. Azadi M, et al. Cost-effectiveness of tuberculosis screening and isoniazid treatment in the TB/HIV in Rio (THRio) Study. Int J Tuberc Lung Dis 2014; 18: 1443-1448. [PubMed: 25517809]

68. Sweeney S, et al. Methodological issues to consider when collecting data to estimate poverty impact in economic evaluations in low-income and middle-income countries. Health Econ 2016; 19: 1300-1317.

69. Trauer JM, et al. The importance of heterogeneity to the epidemiology of tuberculosis. Clin Infect Dis 2019; 69: 159-166. [PubMed: 30383204]

70. Dowdy DW, et al. Heterogeneity in tuberculosis transmission and the role of geographic hotspots in propagating epidemics. Proc Natl Acad Sci USA 2012; 109: 9557-9562. [PubMed: 22645356]

71. Quang Vo LN, et al. Optimizing community screening for tuberculosis: spatial analysis of localized case finding from door-to-door screening for TB in an urban district of Ho Chi Minh City, Viet Nam. PLoS One 2018; 13:12; e0209290. [PubMed: 30562401]

72. Kranzer K, et al. The benefits to communities and individuals of screening for active tuberculosis disease : a systematic review. Int J Tuberc Lung Dis 2013; 17: 432-446. [PubMed: 23485377]

73. World Health Organization. Systematic screening for active tuberculosis: principles and recommendations. WHO/HTM/TB/2013. 04. Geneva, Switzerland: WHO, 2013.

74. MacPherson P, et al. Disparities in access to diagnosis and care in Blantyre, Malawi, identified through enhanced tuberculosis surveillance and spatial analysis. BMC Med 2019; 17:1; 21. [PubMed: 30691470]

75. Auld SC, et al. Where is tuberculosis transmission happening? Insights from the literature, new tools to study transmission and implications for the elimination of tuberculosis. Respirology 2018; 23: 807-817.

76. Oni T, et al. Patterns of HIV, TB, and non-communicable disease multi-morbidity in peri-urban South Africa-a cross sectional study. BMC Infect Dis 2015; 15:1; 1-8. [PubMed: 25567701]

77. Lee EH, et al. Prevalence and impact of diabetes mellitus among patients with active pulmonary tuberculosis in South Korea. Lung 2017; 195: 209-215. [PubMed: 28184994]

78. White LV, et al. Patterns and predictors of co-morbidities in tuberculosis: a cross-sectional study in the Philippines. Sci Rep 2020; 10: 4100. [PubMed: 32139742]

79. Yoon C, et al. Screening for tuberculosis: time to move beyond symptoms. Lancet Respir Med 2019; 7: 202-204. [PubMed: 30823972]

80. Nliwasa M, et al. High mortality and prevalence of HIV and tuberculosis in adults with chronic cough in Malawi: a cohort study. Int J Tuberc Lung Dis 2016; 20: 202-210. [PubMed: 26792472] 
81. Claassens MM, et al. Symptom screening rules to identify active pulmonary tuberculosis: Findings from the Zambian South African Tuberculosis and HIV/AIDS Reduction (ZAMSTAR) trial prevalence surveys. PLoS One 2017; 12:3; e0172881. [PubMed: 28257424]

82. World Health Organization. Chest radiography in tuberculosis detection: summary of current WHO recommendations and guidance on programmatic approaches. WHO/HTM/TB/2016. 20. Geneva, Switzerland: WHO, 2016.

83. UNITAID. Tuberculosis diagnostics technology landscape. 2017

84. Qin ZZ, et al. Using artificial intelligence to read chest radiographs for tuberculosis detection: a multi-site evaluation of the diagnostic accuracy of three deep learning systems (Supplementary Information). Sci Rep 2019; 9:1; 1-10. [PubMed: 30626917]

85. Uplekar M, et al. Programmatic approaches to screening for active tuberculosis. Int J Tuberc Lung Dis 2013; 17: 1248-1256. [PubMed: 24025375]

86. Steingart KR, et al. Xpert@ MTB/RIF assay for pulmonary tuberculosis and rifampicin resistance in adults (Review) Xpert ${ }^{\circledR}$ MTB/RIF assay for pulmonary tuberculosis and rifampicin resistance in adults. Cochrane Database 2014; (1): CD009593.

87. Horne DJ, et al. Xpert MTB/RIF and Xpert MTB/RIF Ultra for pulmonary tuberculosis and rifampicin resistance in adults. Cochrane Database Syst Rev 2019;

88. Codlin AJ, et al. Results from a roving, active case-finding initiative to improve tuberculosis detection among older people in rural cambodia using the Xpert MTB/RIF assay and chest X-ray. J Clin Tuberc Other Mycobact Dis 2018; 13: 22-27. [PubMed: 31720408]

89. Chry M, et al. Can the high sensitivity of Xpert MTB/RIF Ultra be harnessed to save cartridge costs? Results from a pooled sputum evaluation in Cambodia. Trop Med Infect Dis 2020; 5:1; 27.

90. Abdurrahman ST, et al. Testing pooled sputum with Xpert MTB/RIF for diagnosis of pulmonary tuberculosis to increase affordability in low-income countries. J Clin Microbiol 2015; 53: 2502 2508. [PubMed: 26019204] 


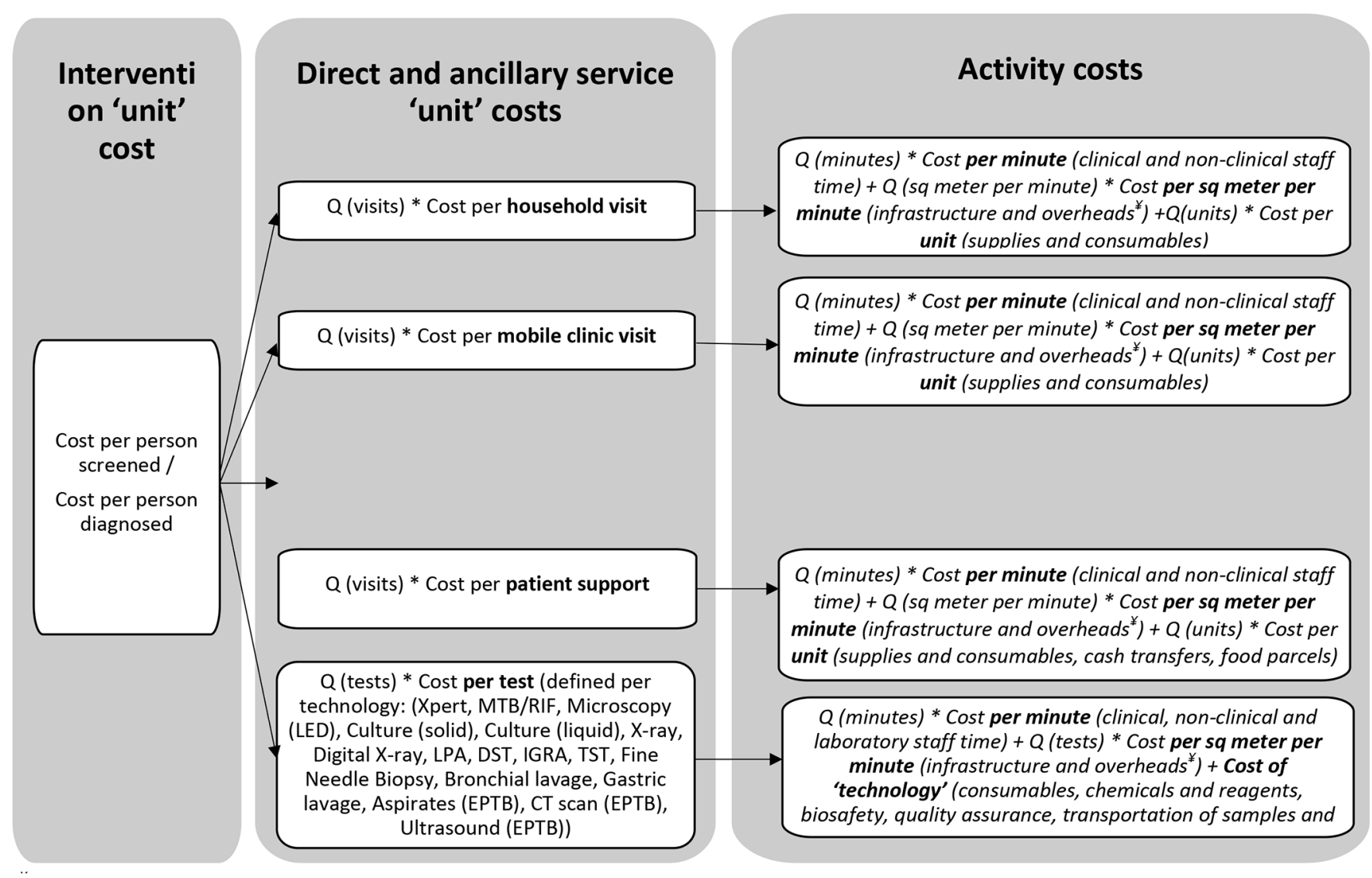

Figure 1.

Example unit cost typology for ACF interventions. ${ }^{\dagger}$ Infrastructure including building, equipment, furniture, maintenance, training; overheads including administration, cleaning, kitchen, utilities, security, laundry, monitoring and evaluation/pharmacovigilance. LED = light-emitting diode; $\mathrm{LPA}=$ line-probe assay; DST = drug susceptibility testing; IGRA = interferon-gamma release assay; $\mathrm{TST}=$ tuberculin skin test EPTB $=$ extrapulmonary $\mathrm{TB}$; $\mathrm{CT}=$ computerized tomography; $\mathrm{ACF}=$ active case-finding . 

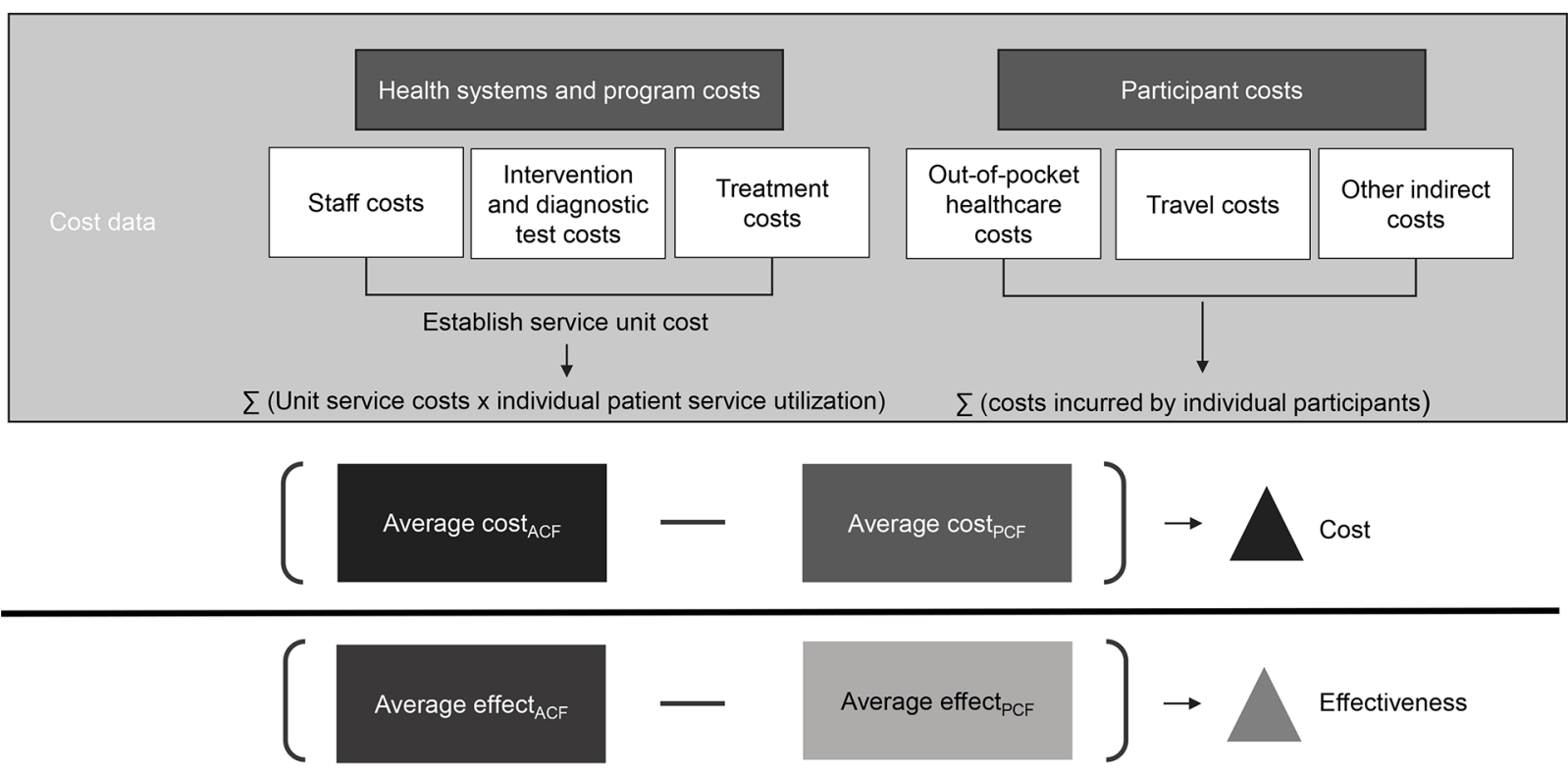

Effectiveness

Figure 2.

The data required to undertake a pragmatic cost-effectiveness analyses for ACF trials.

For each patient enrolled in each arm of the trial, cumulative costs can be tallied based on the frequency of health service use multiplied by corresponding unit costs, including individual patient cost estimates obtained from surveys. The same process is repeated for patient-level effectiveness outcomes. For analysis, total costs and effectiveness estimates can be compared between the intervention groups to compute the ICER.ACF = active case-finding; $\mathrm{PCF}=$ passive case finding; $\mathrm{ICER}=$ incremental cost effectiveness ratio 


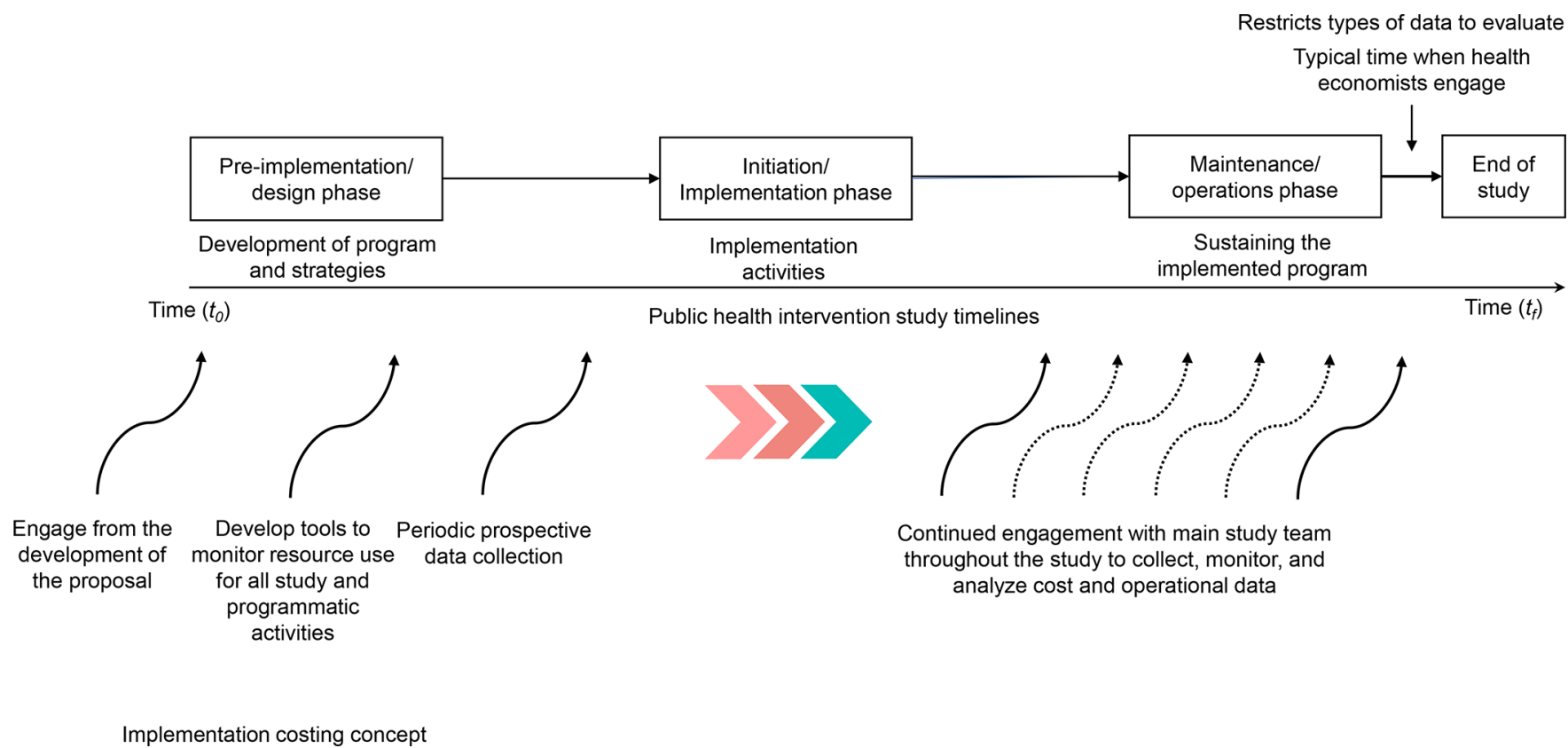

Figure 3.

Promoting early engagement for health economic studies. Prospective assessment of costs and operations of public health interventions: advantages and processes. Typically, health economists are involved late in any study of health interventions; this restricts the types of costs and intervention operations data that can be assessed. In prospective health economic evaluation studies, early engagement of health economists is recommended from the preimplementation/designing phase of the study. Such early engagement enables development and integration of necessary data collection tools that can be used to collect and link cost, operational (study and intervention field operations), and patient relevant data throughout the study. These data can be periodically analyzed to monitor data quality and to aid operations and programmatic management of the intervention. 


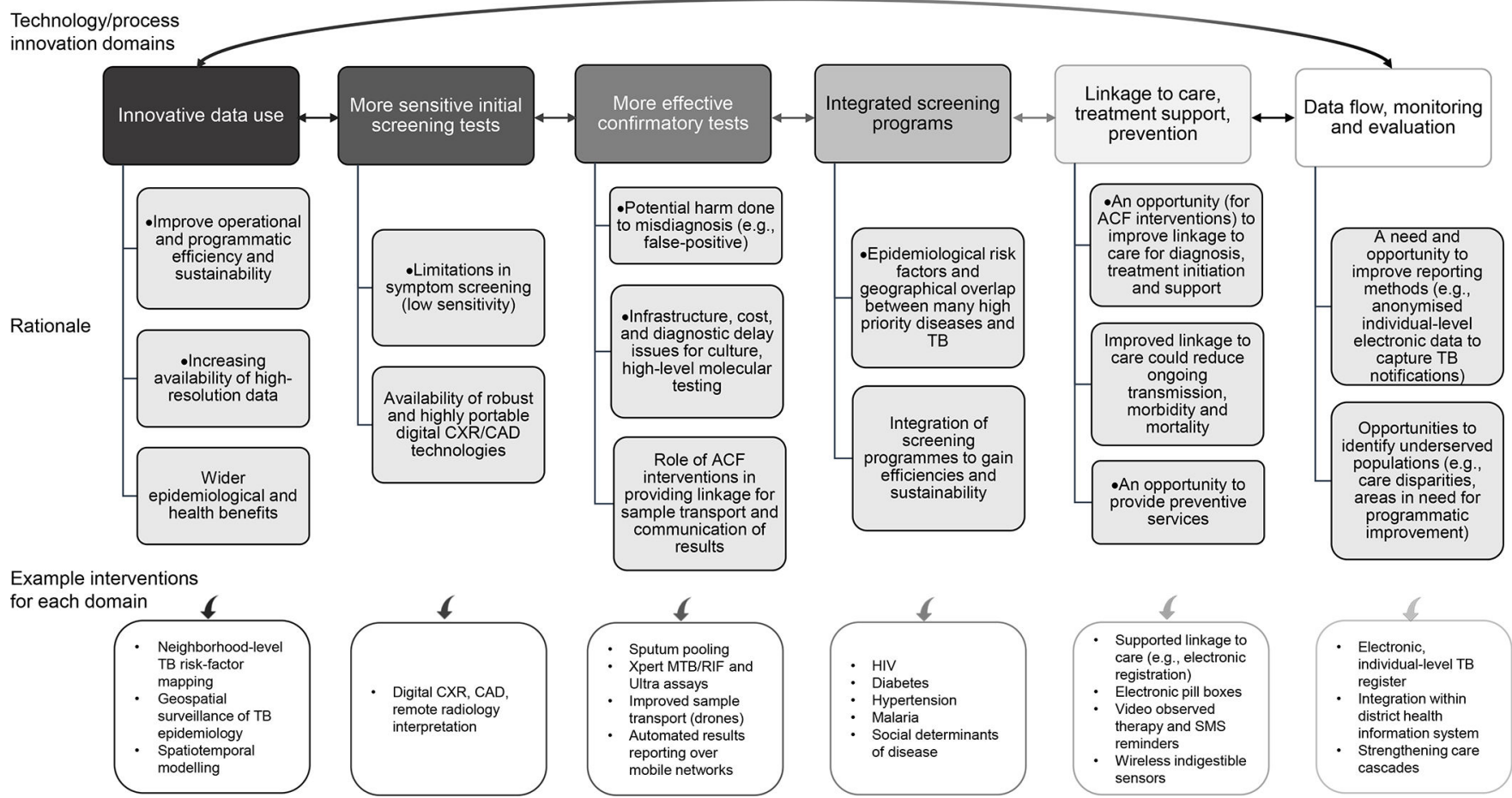

Figure 4.

Technological and process innovations in the implementation and evaluation of tuberculosis active case-finding. The technology and process innovations for TB ACF interventions may be broken down into six distinct domains. The rationale for each technological/ process innovation domain can help improve 1) better targeting of population groups for ACF interventions; 2) screening for patients with presumptive TB; 3) bacteriologic diagnosis of TB; 4) programmatic efficiency; 5) patient linkage to care; and 6) evaluation of intervention performance and impact with adoption/implementation of example interventions/technologies. $\mathrm{CXR}=$ chest $\mathrm{X}$-ray $; \mathrm{CAD}=$ computer-aided design; $\mathrm{ACF}=$ active case-finding. 


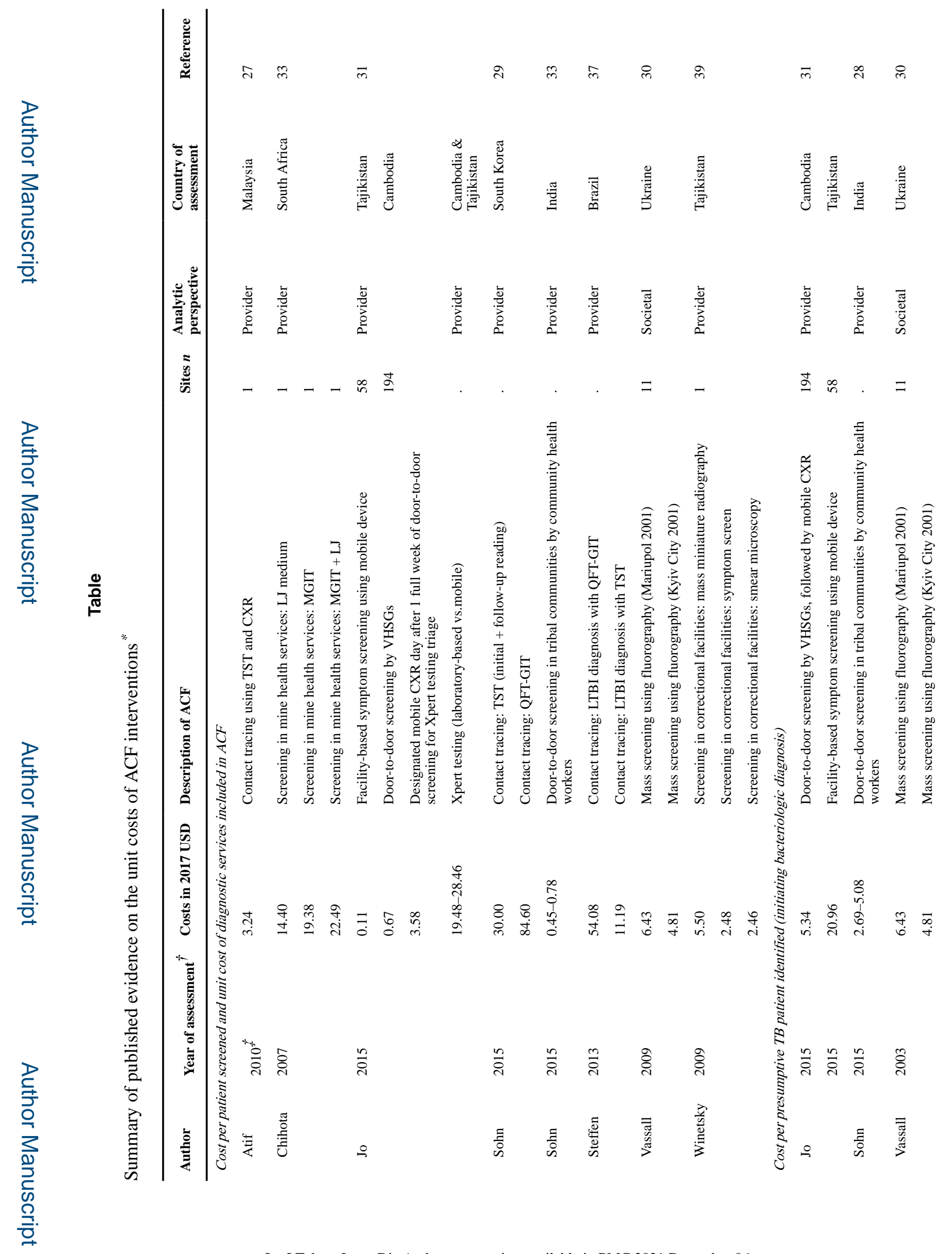

Int J Tuberc Lung Dis. Author manuscript; available in PMC 2021 December 06. 
Sohn et al.

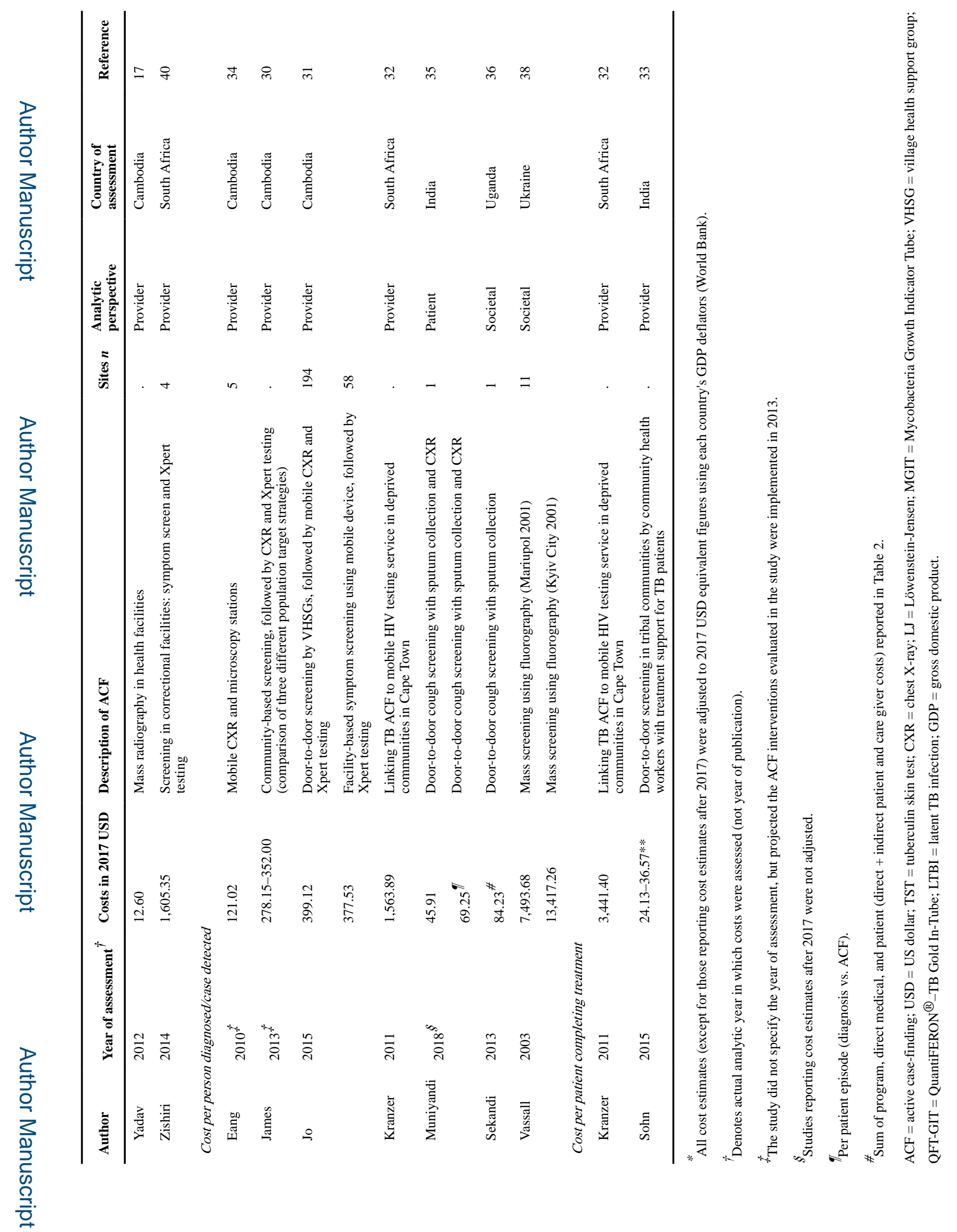

Int J Tuberc Lung Dis. Author manuscript; available in PMC 2021 December 06. 